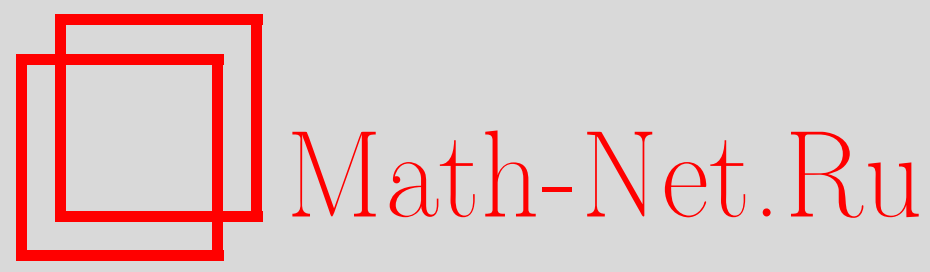

Г. А. Иосифьян, Об усреднении задач теории упругости с краевыми условиями Синьорини, Матем. заметки, 2004, том 75, выпуск 6, 818-833

DOI: https://doi.org/10.4213/mzm73

Использование Общероссийского математического портала Math-Net.Ru подразумевает, что вы прочитали и согласны с пользовательским соглашением http://www . mathnet.ru/rus/agreement

Параметры загрузки:

IP: 54.198 .187 .58

26 апреля 2023 г., 12:58:22 


\title{
ОБ УСРЕДНЕНИИ ЗАДАЧ ТЕОРИИ УПРУГОСТИ С КРАЕВЫМИ УСЛОВИЯМИ СИНЬОРИНИ
}

\section{Г. А. Иосифьян}

\begin{abstract}
В перфорированной области $\Omega^{\varepsilon}=\Omega \cap \varepsilon \omega$, образованной из фиксированной области $\Omega$ и $\varepsilon$-сжатия 1-периодической области $\omega$, рассматриваются задачи теории упругости для вариационных неравенств с краевыми условиями Синьорини на части поверхности перфорации $S_{0}^{\varepsilon}$. Исследуется асимптотическое поведение решений при $\varepsilon \rightarrow 0$ в зависимости от структуры множества $S_{0}^{\varepsilon}$. В общем случае предельная (усредненная) задача имеет два отличительных свойства: (i) предельное множество допустимых перемещений задается нелинейными ограничениями почти всюду в области $\Omega$, т.е. условия Синьорини на поверхности $S_{0}^{\varepsilon}$ в пределе могут превратиться в условия во внутренних точках $\Omega$; (ii) предельная задача формулируется для усредненного лагранжиана, который не обязательн совпадает с квадратичной формой, обычно задающей усредненный тензор упругости. Теоремы об усреднении таких задач получены методом двухмасштабной сходимости. Описана зависимость предельного множества допустимых перемещений и усредненного лагранжиана от геометрии множества $S_{0}^{\varepsilon}$, на котором заданы условия Синьорини.
\end{abstract}

Библиографоия: 12 названий.

Пусть $\Omega^{\varepsilon}=\Omega \cap \varepsilon \omega-$ перфорированная область в $\mathbb{R}^{n}$ с $\varepsilon$-периодической структурой. Здесь $\Omega$ - фиксированная ограниченная область, $\varepsilon>0$ - малый параметр, $\omega-$ 1-периодическая область (т.е. $\omega$ инвариантна относительно любых сдвигов на векторы с целочисленными компонентами), а $\varepsilon \omega$ - ее гомотетическое сжатие. Положим

$$
\square=] 0,1\left[{ }^{n}, \quad \omega_{\square}=\square \cap \omega, \quad \partial_{\square} \omega=\partial \omega \cap\left[0,1\left[{ }^{n} .\right.\right.\right.
$$

Считаем, что $\Omega, \Omega^{\varepsilon}$, а также ячейка периодичности $\omega_{\square}-$ связные открытые множества с липшицевой границей.

Множество $\Omega \backslash \varepsilon \omega$ представляет собой перфорацию области $\Omega$ и может состоять, например, из полостей или сквозных каналов. Границу области $\Omega^{\varepsilon}$ представим в виде $\partial \Omega^{\varepsilon}=\Gamma^{\varepsilon} \cup S^{\varepsilon}$, где $\Gamma^{\varepsilon}=\partial \Omega \cap \partial \Omega^{\varepsilon}-$ внешняя часть гранииы, а $S^{\varepsilon}=\Omega \cap \varepsilon \partial \omega-$ поверхность полостей внутри $\Omega$.

Рассмотрим упругое тело, занимающее область $\Omega^{\varepsilon}$, тензор напряжений которого в точке $x=\left(x_{1}, \ldots, x_{n}\right)$ задается $(n \times n)$-матрицей $\boldsymbol{\sigma}\left(\boldsymbol{u}^{\varepsilon}\right)=\mathcal{A}^{\varepsilon}(x) \boldsymbol{e}\left(\boldsymbol{u}^{\varepsilon}\right)$. Здесь

Работа выполнена при поддержке Российского фонда фундаментальных исследований, гранты № 98-01-00450 и № 00-15-96945. 
$\boldsymbol{u}^{\varepsilon}=\boldsymbol{u}^{\varepsilon}(x)={ }^{t}\left(u_{1}^{\varepsilon}, \ldots, u_{n}^{\varepsilon}\right)-$ вектор перемещений (столбец), $\boldsymbol{e}\left(\boldsymbol{u}^{\varepsilon}\right)=2^{-1}\left(\nabla \boldsymbol{u}^{\varepsilon}+\right.$ $\left.{ }^{t} \nabla \boldsymbol{u}^{\varepsilon}\right)$ - линеаризованны й тензор деформаций, т.е. матрица с элементами $2^{-1} \times$ $\left(\partial u_{i}^{\varepsilon} / \partial x_{j}+\partial u_{j}^{\varepsilon} / \partial x_{i}\right) ; \mathcal{A}^{\varepsilon}(x)-$ тензор упругости, представляющий собой линейноепреобразование в пространстве $\mathbb{M}^{n}$ вешественных $(n \times n)$-матриц и имеющий вид

$$
\mathcal{A}^{\varepsilon}(x)=\mathcal{A}\left(\varepsilon^{-1} x\right)=\left\{a_{i j}^{h k}\left(\varepsilon^{-1} x\right)\right\}, \quad(\mathcal{A}(\xi) \boldsymbol{p})_{j k}=a_{i j}^{h k}(\xi) p_{i h} \quad \forall \boldsymbol{p}=\left\{p_{i h}\right\} \in \mathbb{M}^{n} .
$$

Коэффициенты $a_{i j}^{h k}(\xi)$ являются ограниченными измеримьми 1-периодическими функциями $\xi \in \mathbb{R}^{n}$, удовлетворяющими принятым в теории упругости условиям симметрии и положительной определенности, которые можно записать в виде (см. $[1$, лемма 6$])$

$$
\left.\begin{array}{c}
\boldsymbol{q}: \mathcal{A}(\xi) \boldsymbol{p}=\boldsymbol{p}: \mathcal{A}(\xi) \boldsymbol{q} \quad \forall \boldsymbol{p}, \boldsymbol{q} \in \mathbb{M}^{n}, \\
\mathcal{A}(\xi) \boldsymbol{p}=\mathbf{0} \Longleftrightarrow{ }^{t} \boldsymbol{p}=-\boldsymbol{p}, \\
: \mathcal{A}(\xi) \boldsymbol{p} \leqslant \kappa_{2}|\boldsymbol{p}|^{2} \quad \forall \boldsymbol{p} \in \mathbb{S}^{n} \quad\left(\kappa_{1}, \kappa_{2}=\text { const }>0\right),
\end{array}\right\}
$$

где ${ }^{t} \boldsymbol{p}$ - матрища, транспонированная к $\boldsymbol{p} \in \mathbb{M}^{n} ; \mathbb{S}^{n}$ - пространство вещественных симметрических $(n \times n)$-матрищ; $\boldsymbol{p}: \boldsymbol{q}=p_{i j} q_{i j}$ - скалярное произведение матриц, $|\boldsymbol{p}|^{2}=$ $\boldsymbol{p}: \boldsymbol{p}$. Здесь и далее предполагается суммирование по повторяющимся латинским индексам. Полужирньм шрифоом обозначаются матрицы и векторы (столбцы); $\boldsymbol{\eta} \cdot \boldsymbol{\zeta}=\eta_{i} \zeta_{i}$ - скалярное произведение векторов $\boldsymbol{\eta}, \boldsymbol{\zeta} \in \mathbb{R}^{n}$.

Введем следующие функциональные пространства. Для ограниченной липшицевой области $Q$ пространство $H^{1}(Q)$ есть пополнение $C^{\infty}(\bar{Q})$ по норме

$$
\|u\|_{1, Q}=\|u\|_{L^{2}(Q)}+\|\nabla u\|_{L^{2}(Q)} .
$$

Нормы в пространствах вектор-функций $H^{1}(Q)^{N}$ и $L^{2}(Q)^{N}$ обозначаются также через $\|\cdot\|_{1, Q}$ и $\|\cdot\|_{0, Q}$ соответственно.

Для множества $\gamma \subset \partial Q$, meas $_{n-1} \gamma \neq 0$, введем пространство $H_{0}^{1}(Q, \gamma)$, состоящее из всех $u \in H^{1}(Q)$ с нулевым следом на $\gamma ; H_{0}^{1}(Q, \partial Q)=H_{0}^{1}(Q)$.

Пространство 1-периодических функций $H_{\text {per }}^{1}(\omega)$ есть пополнение $C_{\text {per }}^{\infty}(\bar{\omega})$ по норме $\|\cdot\|_{1, \omega_{\square}}$. Для $v \in H_{\mathrm{per}}^{1}(\omega)$ определим среднее значение

$$
\langle v\rangle_{\omega}=\left|\omega_{\square}\right|^{-1} \int_{\omega_{\square}} v(\xi) d \xi, \quad \text { где }\left|\omega_{\square}\right|=\operatorname{meas} \omega_{\square} .
$$

Наконец, $L_{\text {per }}^{p}(\square)$ и $H_{\text {per }}^{1}(\square)$ - соответствующие пространства 1-периодических функций в $\mathbb{R}^{n}$.

На поверхности полостей $S^{\varepsilon}$ рассмотрим множество $S_{0}^{\varepsilon}=S^{\varepsilon} \cap \varepsilon \partial_{0} \omega$, где $\partial_{0} \omega$ - измеримое 1-периодическое подмножество $\partial \omega$. Для задания условий Синьорини на $S_{0}^{\varepsilon}$ и нулевых условий Дирихле на внешней части границы $\Gamma^{\varepsilon}$ введем множество допустимых перемещений

$$
\mathcal{V}^{\varepsilon}=\left\{\boldsymbol{v} \in H_{0}^{1}\left(\Omega^{\varepsilon}, \Gamma^{\varepsilon}\right)^{n}: \boldsymbol{v} \cdot \boldsymbol{\nu}^{\varepsilon} \leqslant 0 \text { п.в. на } S_{0}^{\varepsilon}\right\},
$$

где $\boldsymbol{\nu}^{\varepsilon}$ - единичньй вектор внешней нормали к $\partial \Omega^{\varepsilon}$.

Будем изучать следующую задачу о равновесии упругого тела, жестко закрепленного вдоль $\Gamma^{\varepsilon}$, а на $S_{0}^{\varepsilon}$ имеющего односторонний контакт с препятствием без трения (см., например, [2]). 
ЗАДАчА. Для заданных объемных сил $\boldsymbol{f} \in L^{2}(\Omega)^{n}$ найти перемещение $\boldsymbol{u}^{\varepsilon} \in \mathcal{V}^{\varepsilon}$, удовлетворяющее вариационному неравенству

$$
\int_{\Omega^{\varepsilon}} \boldsymbol{e}\left(\boldsymbol{v}-\boldsymbol{u}^{\varepsilon}\right): \mathcal{A}\left(\varepsilon^{-1} x\right) \boldsymbol{e}\left(\boldsymbol{u}^{\varepsilon}\right) d x \geqslant \int_{\Omega^{\varepsilon}} \boldsymbol{f} \cdot\left(\boldsymbol{v}-\boldsymbol{u}^{\varepsilon}\right) d x \quad \forall \boldsymbol{v} \in \mathcal{V}^{\varepsilon} .
$$

Существование и единственность решения этой задачи устанавливаются обычными вариационными методами (см. [2], [3]), поскольку для элементов $H^{1}\left(\Omega^{\varepsilon}, \Gamma^{\varepsilon}\right)^{n}$ выполнено неравенство Корна (см. [4], [5]), а $\mathcal{V}^{\varepsilon}$ является замкнутым выпуклым конусом в $H^{1}\left(\Omega^{\varepsilon}, \Gamma^{\varepsilon}\right)^{n}$.

Наша цель - описать предельное поведение решений $\boldsymbol{u}^{\varepsilon}$ при $\varepsilon \rightarrow 0$ и выяснить, как оно зависит от геометрической структуры множества $S_{0}^{\varepsilon}$, на котором заданы условия Синьорини.

Поскольку $\partial \omega$ - липшицева поверхность, то единичная внешняя нормаль $\nu(\xi)$ к $\partial \omega$ существует при почти всех $\xi \in \partial \omega$ и можно рассмотреть полупространства

$$
\mathcal{M}_{\xi}=\left\{\boldsymbol{\eta} \in \mathbb{R}^{n}: \boldsymbol{\eta} \cdot \boldsymbol{\nu}(\xi) \leqslant 0\right\}=\left\{\boldsymbol{\eta} \in \mathbb{R}^{n}:(\boldsymbol{\eta} \cdot \boldsymbol{\nu}(\xi))^{+}=0\right\},
$$

где $a^{+}=\max \{0, a\}$. Легко видеть, что краевые условия Синьорини $\boldsymbol{v} \cdot \boldsymbol{\nu}^{\varepsilon} \leqslant 0$ на $S_{0}^{\varepsilon}$ в (2) можно записать в эквивалентном виде

$$
\left.\boldsymbol{v}(x)\right|_{S_{0}^{\varepsilon}} \in \mathcal{M}_{\varepsilon^{-1} x} \Longleftrightarrow \int_{S_{0}^{\varepsilon}}\left|\left(\boldsymbol{v} \cdot \boldsymbol{\nu}\left(\varepsilon^{-1} x\right)\right)^{+}\right|^{2} d S=0 .
$$

Чтобы для задачи (3) сформулировать предельную задачу (называемую также усредненной) и описать предельное множество допустимых перемещений, введем следующие замкнутые выпуклые конусы в пространствах $\mathbb{R}^{n}$ и $H_{0}^{1}(\Omega)^{n}$ :

$$
\begin{gathered}
\mathcal{M}^{0}=\left\{\boldsymbol{\eta} \in \mathbb{R}^{n}: \int_{\sigma_{0}}\left|(\boldsymbol{\eta}, \boldsymbol{\nu}(\xi))^{+}\right|^{2} d S_{\xi}=0\right\}, \quad \sigma_{0}=\partial_{0} \omega \cap\left[0,1\left[^{n},\right.\right. \\
\mathcal{V}^{0}=\left\{\boldsymbol{v} \in H_{0}^{1}(\Omega)^{n}: \boldsymbol{v}(x) \in \mathcal{M}^{0} \text { п.в. в } \Omega\right\} .
\end{gathered}
$$

Если $\partial_{0} \omega-$ непустое открытое множество на $\partial \omega$ и $\boldsymbol{\nu}(\xi)$ непрерывна на $\partial_{0} \omega$, то очевидно, что

$$
\mathcal{M}^{0}=\bigcap_{\xi \in \partial_{0} \omega} \mathcal{M}_{\xi}=\bigcap_{\xi \in \sigma_{0}} \mathcal{M}_{\xi}
$$

Не ограничивая общности, можем считать, что конусы (5) и (7) совпадают, поскольку мы можем устранить из $\partial_{0} \omega$ множество нулевой меры на $\partial \omega$, не меняя при этом условий Синьорини на $S_{0}^{\varepsilon}$, которые вьполнены для следов допустимых перемещений почти всюду.

Рассмотрим следующие два типа условий Синьорини на $S_{0}^{\varepsilon}=\Omega \cap \varepsilon \partial_{0} \omega$ в соответствии с геометрической структурой множества $\partial_{0} \omega$.

Будем говорить, что условия Синьорини на $S_{0}^{\varepsilon}$ оставляют $n$ направлений выхода, если $\operatorname{dim} \mathcal{M}^{0}=n$ (под размерностью конуса в $\mathbb{R}^{n}$ имеется в виду размерность его 

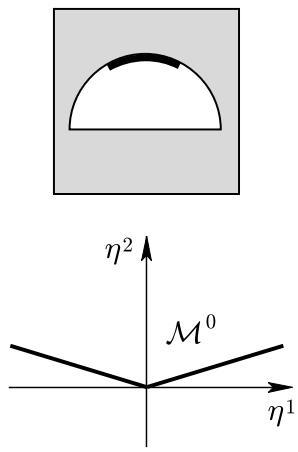

(a)
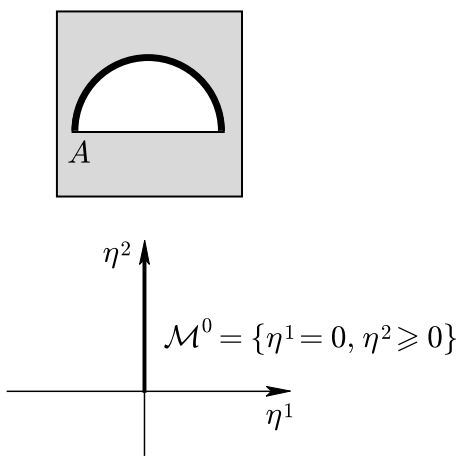

(c)
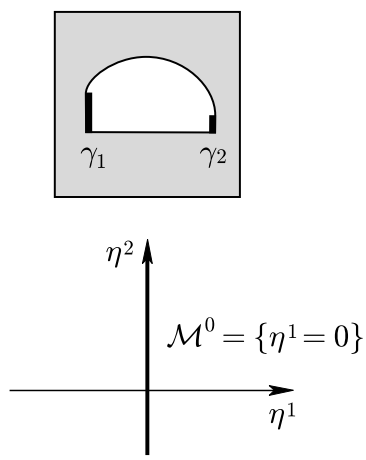

(b)
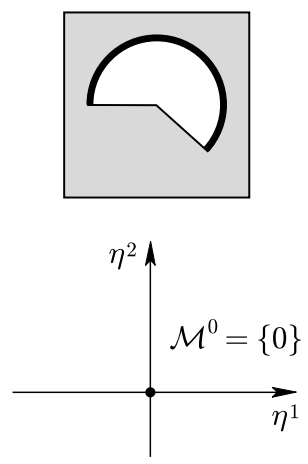

(d)

Рис. 1. Условия Синьорини заданы на множествах, прилегающих к жирным линиям

линейной оболочки), что эквивалентно наличию у конуса $\mathcal{M}^{0}$ внутренних точек в топологии $\mathbb{R}^{n}$. Например, при $n=2$ такими являются условия Синьорини на рис. 1 (a), заданные на дуге окружности, раствор которой строго меньше $2 \pi$.

Будем говорить, что условия Синьорини на $S_{0}^{\varepsilon}$ задают двухсторонние препятствия nо $n_{0}$ направлениям, $0<n_{0} \leqslant n$, если единичная внешняя нормаль $\nu(\xi)$ на $\partial_{0} \omega$ есть столбец вида $\boldsymbol{\nu}(\xi)={ }^{t}(\widehat{\boldsymbol{\nu}}(\xi), \widetilde{0})$, где $\widehat{\boldsymbol{\nu}}(\xi)={ }^{t}\left(\nu_{1}, \ldots, \nu_{n_{0}}\right) \in \mathbb{R}^{n_{0}}$ (при $n_{0}=n$ полагаем $\widehat{\nu}=\nu)$ и

$$
\widehat{\mathcal{M}}^{0}=\left\{\widehat{\boldsymbol{\eta}} \in \mathbb{R}^{n_{0}}: \int_{\sigma_{0}}\left|(\widehat{\boldsymbol{\eta}} \cdot \widehat{\boldsymbol{\nu}})^{+}\right|^{2} d S=0\right\}=\{\mathbf{0}\}
$$

Очевидно, в этом случае $0 \leqslant \operatorname{dim} \mathcal{M}^{0}<n$. Пример таких краевых условий при $n=2$, $n_{0}=1$ показан на рис. 1 (b), где множество $\sigma_{0}$ состоит из двух параллельных отрезков $\gamma_{1}, \gamma_{2}$; еще один пример дан на рис. $1(\mathrm{~d})$, когда $n_{0}=n=2$, а $\sigma_{0}$ - дуга окружности раствором, строго большим $2 \pi$.

Случай условий Синьорини, оставляющих $n$ направлений выхода, рассмотрен в [1], где показано (при некоторых несущественных дополнительных условиях на структуру ячейки $\left.\omega_{\square}\right)$, что решения $\boldsymbol{u}^{\varepsilon}$ задачи (3) в определенном смысле близки к решению $\boldsymbol{u}^{0}$ 
усредненной задачи для вариационного неравенства

$$
\boldsymbol{u}^{0} \in \mathcal{V}^{0}, \quad \int_{\Omega} \boldsymbol{e}\left(\boldsymbol{u}^{0}\right): \widehat{\mathcal{A}} \boldsymbol{e}\left(\boldsymbol{v}-\boldsymbol{u}^{0}\right) d x \geqslant \int_{\Omega} \boldsymbol{f} \cdot\left(\boldsymbol{v}-\boldsymbol{u}^{0}\right) d x \quad \forall \boldsymbol{v} \in \mathcal{V}^{0}
$$

Здесь $\widehat{\mathcal{A}}$ - обычньй усредненньй тензор упругости (см. [4], [6]), определяемый из линейной 1-периодической краевой задачи в области $\omega$ (задачи на ячейке), которая совпадает с уравнением Эйлера для следующей задачи на минимум (с параметром $\boldsymbol{p})$ :

$$
\mathbb{A}(\boldsymbol{p})=\inf _{\boldsymbol{v} \in \mathbb{V}}\left\langle\left(\boldsymbol{e}_{\xi}(\boldsymbol{v})+\boldsymbol{p}\right): \mathcal{A}(\xi)\left(\boldsymbol{e}_{\xi}(\boldsymbol{v})+\boldsymbol{p}\right)\right\rangle_{\omega}, \quad \boldsymbol{p} \in \mathbb{M}^{n}
$$

где $\mathbb{V}=H_{\text {per }}^{1}(\omega)^{n}, \boldsymbol{e}_{\xi}(\boldsymbol{v})=2^{-1}\left(\nabla_{\xi} \boldsymbol{v}(\xi)+{ }^{t} \nabla_{\xi} \boldsymbol{v}(\xi)\right)$. Для любой матрицы $\boldsymbol{p} \in \mathbb{M}^{n}$ решение $\boldsymbol{U}(\boldsymbol{p}, \xi)$ задачи (9), определенное с точностью до постоянного слагаемого, можно выбрать так, чтобы оно было линейной функцией от $\boldsymbol{p}$ со значениями в $H_{\mathrm{per}}^{1}(\omega)^{n}$. Функция $\mathbb{A}(\boldsymbol{p})$, называемая усредненным лагранжианом, в рассматриваемом случае является квадратичной формой по $\boldsymbol{p}$, а усредненньй тензор $\widehat{\mathcal{A}}$ определяется соотношениями

$$
\boldsymbol{p}: \widehat{\mathcal{A}} \boldsymbol{p}=\mathbb{A}(\boldsymbol{p}) \quad \text { или } \quad \widehat{\mathcal{A}} \boldsymbol{p}=\left\langle\mathcal{A}(\xi)\left(\boldsymbol{e}_{\xi}(\boldsymbol{U}(\boldsymbol{p}, \xi))+\boldsymbol{p}\right)\right\rangle_{\omega} \forall \boldsymbol{p} \in \mathbb{M}^{n}
$$

Таким образом [1], при наличии $n$ направлений выхода (т.е. при $\operatorname{dim} \mathcal{M}^{0}=n$ ) условия Синьорини на поверхностях $S_{0}^{\varepsilon}$ в пределе переходят в односторонние ограничения (6) почти всюду в области $\Omega$, но усредненный тензор упругости остается тем же, что и для линейной задачи с нулевыми условиями Неймана на перфорации.

В настоящей работе изучаются различные случаи усреднения задач с условиями Синьорини на $S_{0}^{\varepsilon}$, для которых $\operatorname{dim} \mathcal{M}^{0}<n$. Для этого воспользуемся методом двухмасштабной сходимости, предварительно напомнив ее определение и основные свойства (см. [7]-[9]).

Пусть $L^{2}(\Omega, H)$ - лебегово пространство классов функций, заданных при почти всех $x \in \Omega$ и принимающих значения в сепарабельном гильбертовом пространстве $H, \mathrm{a}$ $C(\bar{\Omega}, H)$ - пространство непрерьвных в $\bar{\Omega}$ функций со значениями в $H$. В дальнейшем $H$ будет соболевским или лебеговым пространством, элементами которого являются 1-периодические функции аргумента $\xi \in \omega$, а элементы $\boldsymbol{u}, \boldsymbol{v} \in L^{2}(\Omega, H)$ будем часто записьвать в виде $\boldsymbol{u}(x, \xi), \boldsymbol{v}(y, \xi)$, где $x, y \in \Omega, \xi \in \omega$.

Символы $\rightarrow, \rightarrow$ и $\stackrel{2}{\rightarrow}$ соответственно обозначают сильную, слабую и двухмасштабную сходимости. В зависимости от контекста $\varepsilon$ может обозначать как рассматриваемую последовательность, так и ее подпоследовательность. Для постоянньх в неравенствах зависимость от $\varepsilon$ указьвается в явном виде, а постоянные $c, c_{i}, C_{j}$ и т.п. не зависят от $\varepsilon$.

Будем говорить, что последовательность $u^{\varepsilon} \in L^{2}(\Omega)$ двухмасштабно сходится $\kappa u^{0}(x, \xi) \in L^{2}\left(\Omega, L_{\mathrm{per}}^{2}(\square)\right)\left(\right.$ короче, $\left.u^{\varepsilon}(x) \stackrel{2}{\rightarrow} u^{0}(x, \xi)\right)$, если для любых $\varphi(x) \in C(\bar{\Omega})$ и $\psi(\xi) \in L_{\text {per }}^{2}(\square)$ имеет место сходимость

$$
\int_{\Omega} u^{\varepsilon}(x) \varphi(x) \psi\left(\varepsilon^{-1} x\right) d x \rightarrow \int_{\Omega \times \square} u^{0}(x, \xi) \varphi(x) \psi(\xi) d x d \xi \quad \text { при } \varepsilon \rightarrow 0 \text {. }
$$


СвоЙСтва компАктности. (i) Пусть $u^{\varepsilon} \in L^{2}(\Omega), \sup _{\varepsilon}\left\|u^{\varepsilon}\right\|_{0, \Omega}<\infty$. Тогда имеет место следующая сходимость по подпоследовательности:

$$
u^{\varepsilon}(x) \stackrel{2}{\rightarrow} u^{0}(x, \xi), \quad u^{0}(x, \xi) \in L^{2}\left(\Omega, L_{\mathrm{per}}^{2}(\square)\right) .
$$

(ii) Пусть $u^{\varepsilon} \in H^{1}(\Omega), \sup _{\varepsilon}\left\|u^{\varepsilon}\right\|_{1, \Omega}<\infty$. Тогда найдymся $u^{0} \in H^{1}(\Omega) u$ $u^{1}(x, \xi) \in L^{2}\left(\Omega, H_{\mathrm{per}}^{1}(\square) / \mathbb{R}^{1}\right)$ такие, ито имеет место следующая сходимость по подпоследовательности:

$$
u^{\varepsilon}(x) \stackrel{L^{2}(\Omega)}{\rightarrow} u^{0}(x), \quad \nabla_{x} u^{\varepsilon} \stackrel{2}{\rightarrow} \nabla_{x} u^{0}(x)+\nabla_{\xi} u^{1}(x, \xi) .
$$

(iii) Пусть $u^{\varepsilon} \in H^{1}(\Omega), \sup _{\varepsilon}\left\|\varepsilon^{-1} u^{\varepsilon}\right\|_{0, \Omega}+\sup _{\varepsilon}\left\|\nabla u^{\varepsilon}\right\|_{0, \Omega}<\infty$. Тогда найдется $u^{1}(x, \xi) \in L^{2}\left(\Omega, H_{\mathrm{per}}^{1}(\square)\right)$ такое, что имеет место следующая сходимость для подпоследовательности:

$$
\varepsilon^{-1} u^{\varepsilon}(x) \stackrel{2}{\rightarrow} u^{1}(x, \xi), \quad \nabla_{x} u^{\varepsilon} \stackrel{2}{\rightarrow} \nabla_{\xi} u^{1}(x, \xi) .
$$

СвоЙСТвО СРЕДНЕГО ЗНАчЕНИЯ. Для любы $\varphi(x) \in C(\bar{\Omega}) u \psi(\xi) \in L_{\mathrm{per}}^{1}(\square)$

$$
\lim _{\varepsilon \rightarrow 0} \int_{\Omega} \varphi(x) \psi\left(\varepsilon^{-1} x\right) d x=\int_{\Omega \times \square} \varphi(x) \psi(\xi) d x d \xi .
$$

НЕРАВЕНСТВО ТИПА ПОЛУНЕПРЕРЫВНОСТИ СНИЗУ. Пусть $\boldsymbol{M}(\xi) \geqslant 0$ - 1-nерuодuческая $(N \times N)$-матрича с ограниченными измеримыми әлементами, $а \boldsymbol{V}^{\varepsilon} \in$ $L^{2}(\Omega)^{N}$ - последовательность векторов, двухмасштабно сходящаяся $\kappa \boldsymbol{V}^{0} \in L^{2}(\Omega$, $\left.L_{\text {per }}^{2}(\square)^{N}\right)$. Toгдa

$$
\liminf _{\varepsilon \rightarrow 0} \int_{\Omega} \boldsymbol{V}^{\varepsilon}(x) \cdot \boldsymbol{M}\left(\varepsilon^{-1} x\right) \boldsymbol{V}^{\varepsilon}(x) d x \geqslant \int_{\Omega \times \square} \boldsymbol{V}^{0}(x, \xi) \cdot \boldsymbol{M}(\xi) \boldsymbol{V}^{0}(x, \xi) d x d \xi .
$$

ДокАЗАТЕЛЬСТВА свойств компактности и среднего значения даны, например, в [9]. Приведем краткий вьвод неравенства (13). Пусть $\Omega_{1}, \ldots, \Omega_{m}, m<\infty,-$ произвольные липшицевы подобласти $\Omega$ такие, что $\Omega_{i} \cap \Omega_{j}=\varnothing$ при $i \neq j$, и пусть

$$
\boldsymbol{\Phi}(x, \xi)=\sum_{i=1}^{m} \varphi_{i}(x) \boldsymbol{\psi}_{i}(\xi), \quad 0 \leqslant \varphi_{i} \in C_{0}^{\infty}(\Omega), \quad \operatorname{supp} \varphi_{i} \in \Omega_{i}, \quad \boldsymbol{\psi}_{i} \in L_{\mathrm{per}}^{2}(\square)^{N} .
$$

Из очевидного неравенства

$$
0 \leqslant \int_{\Omega}\left(\boldsymbol{V}^{\varepsilon}-\boldsymbol{\Phi}\left(x, \varepsilon^{-1} x\right)\right) \cdot \boldsymbol{M}\left(\varepsilon^{-1} x\right)\left(\boldsymbol{V}^{\varepsilon}-\boldsymbol{\Phi}\left(x, \varepsilon^{-1} x\right)\right) d x,
$$

пользуясь свойством среднего значения и двухмасштабной сходимостью $\boldsymbol{V}^{\varepsilon}(x)$, выводим

$$
\begin{aligned}
& \liminf _{\varepsilon \rightarrow 0} \int_{\Omega} \boldsymbol{V}^{\varepsilon} \cdot \boldsymbol{M}\left(\varepsilon^{-1} x\right) \boldsymbol{V}^{\varepsilon} d x \geqslant-\int_{\Omega \times \square} \boldsymbol{\Phi}(x, \xi) \cdot \boldsymbol{M}(\xi) \boldsymbol{\Phi}(x, \xi) d x d \xi \\
& \quad+\int_{\Omega \times \square} \boldsymbol{V}^{0}(x, \xi) \cdot \boldsymbol{M}(\xi) \boldsymbol{\Phi}(x, \xi) d x d \xi+\int_{\Omega \times \square} \boldsymbol{\Phi}(x, \xi) \cdot \boldsymbol{M}(\xi) \boldsymbol{V}^{0}(x, \xi) d x .
\end{aligned}
$$

Поскольку функции $\boldsymbol{\Phi}(x, \xi)$ указанной структуры образуют всюду плотное множество в $L^{2}\left(\Omega, L_{\text {per }}^{2}(\square)^{N}\right)$, то это неравенство верно для $\Phi \equiv \boldsymbol{V}^{0}$, откуда и следует (13).

Покажем теперь, что при двухмасштабной сходимости можно говорить о сохранении краевых условий Синьорини, задаюших двухсторонние препятствия по $n_{0}$ направленияM. 
Лемма 1. Предположим, что условия Синьорини на $S_{0}^{\varepsilon}$ задают двухсторонние препятствия по $n_{0}$ направлениям, $0<n_{0} \leqslant n$, a $\boldsymbol{v}^{\varepsilon} \in H^{1}(\Omega)^{n_{0}}$ - ограниченная последовательность такая, что $\left.\boldsymbol{v}^{\varepsilon} \cdot \widehat{\boldsymbol{\nu}}\left(\varepsilon^{-1} x\right)\right|_{S_{0}^{\varepsilon}} \leqslant 0$. Тогда найдется әлемент $\boldsymbol{v}^{1}(x, \xi) \in L^{2}\left(\Omega, H_{\text {per }}^{1}(\square)^{n_{0}}\right)$ такой, что $\left.\boldsymbol{v}^{1}(x, \xi) \cdot \widehat{\nu}(\xi)\right|_{\xi \in \sigma_{0}} \leqslant 0$ u по подпоследовательности

$$
\varepsilon^{-1} \boldsymbol{v}^{\varepsilon} \stackrel{2}{\rightarrow} \boldsymbol{v}^{1}(x, \xi), \quad \nabla_{x} \boldsymbol{v}^{\varepsilon} \stackrel{2}{\rightarrow} \nabla_{\xi} \boldsymbol{v}^{1}(x, \xi) .
$$

ДокАЗАТЕЛьСтво. Сначала установим равномерные неравенства типа Фридрихса

$$
\begin{gathered}
\|\boldsymbol{v}\|_{0, \square_{k}}^{2} \leqslant C_{k}\left\|\nabla_{\xi} \boldsymbol{v}\right\|_{0, \square_{k}}^{2} \quad \forall \boldsymbol{v}(\xi) \in H^{1}\left(\square_{k}\right)^{n_{0}},\left.\quad \boldsymbol{v}\right|_{\sigma_{0}} \cdot \widehat{\boldsymbol{\nu}}(\xi) \leqslant 0, \\
\left\|\boldsymbol{v}^{\varepsilon}\right\|_{0, \Omega}^{2} \leqslant C \varepsilon^{2}\left\|\nabla_{x} \boldsymbol{v}^{\varepsilon}\right\|_{0, \Omega}^{2} \quad \forall \boldsymbol{v}^{\varepsilon}(x) \in H^{1}(\Omega)^{n_{0}},\left.\quad \boldsymbol{v}^{\varepsilon}\right|_{S_{0}^{\varepsilon}} \cdot \widehat{\boldsymbol{\nu}}\left(\varepsilon^{-1} x\right) \leqslant 0,
\end{gathered}
$$

где $\left.\square_{k}=\right]-k, k\left[{ }^{n}, k \geqslant 1\right.$. Допустим, что (15) не верно. Тогда найдется последовательность $\boldsymbol{w}^{m}(\xi) \in H^{1}\left(\square_{k}\right)^{n_{0}}$, для которой

$$
\left.\boldsymbol{w}^{m}\right|_{\sigma_{0}} \cdot \widehat{\boldsymbol{\nu}}(\xi) \leqslant 0, \quad\left\|\boldsymbol{w}^{m}\right\|_{0, \square_{k}}=1, \quad\left\|\nabla_{\xi} \boldsymbol{w}^{m}\right\|_{0, \square_{k}} \rightarrow 0 \text { при } m \rightarrow \infty .
$$

В силу неравенства Пуанкаре и компактности вложения $H^{1}\left(\square_{k}\right) \subset L^{2}\left(\square_{k}\right)$ найдутся постоянньй вектор $\boldsymbol{\eta}^{0} \in \mathbb{R}^{n_{0}}$ и подпоследовательность $m^{\prime} \rightarrow \infty$ такие, что

$$
\left\|\boldsymbol{w}^{m^{\prime}}-\boldsymbol{\eta}^{0}\right\|_{1, \square_{k}} \rightarrow 0, \quad\left\|\boldsymbol{\eta}^{0}\right\|_{0, \square_{k}}=1 .
$$

По теореме о следе имеем $\boldsymbol{\eta}^{0} \cdot \widehat{\boldsymbol{\nu}}(\xi) \leqslant 0$ п.в. на $\sigma_{0}$ и, следовательно, $\boldsymbol{\eta}^{0} \in \widehat{\mathcal{M}}^{0}$, что противоречит условию $\widehat{\mathcal{M}}^{0}=\{\boldsymbol{0}\}$. Тем самым, (15) доказано.

Рассмотрим куб $Q \supset \bar{\Omega}$ и линейный оператор продолжения $P: H^{1}(\Omega)^{n_{0}} \rightarrow H^{1}(Q)^{n_{0}}$, для которого

$$
\|P \boldsymbol{u}\|_{0, Q} \leqslant c\|\boldsymbol{u}\|_{0, \Omega}, \quad\|P u\|_{1, Q} \leqslant c\|\boldsymbol{u}\|_{1, \Omega}
$$

с постоянной $c$, не зависящей от $\boldsymbol{u}$. Покроем $Q$ малыми $\varepsilon$-кубами вида $\varepsilon\left(z+\square_{k}\right)$ так, чтобы точки $z$ имели целочисленные координаты, а число $\varepsilon$-кубов, пересекающихся с любым заданным $\varepsilon$-кубом, не зависело от $\varepsilon$. Неравенство (16) легко устанавливается применением к $P \boldsymbol{v}^{\varepsilon}$ в каждом из $\varepsilon$-кубов, покрывающих $\Omega$, оценки, которая получается из (15) заменой переменных $\xi=\varepsilon^{-1} x$.

В силу неравенства (16) и свойства компактности (iii) (см. (11)) из ограниченной последовательности

$$
\boldsymbol{v}^{\varepsilon} \in H^{1}(\Omega)^{n_{0}},\left.\quad \boldsymbol{v}^{\varepsilon} \cdot \widehat{\boldsymbol{\nu}}\left(\varepsilon^{-1} x\right)\right|_{S_{0}^{\varepsilon}} \leqslant 0,
$$

можно выделить подпоследовательность, для которой имеет место сходимость (14). Остается показать, что $\left.\boldsymbol{v}^{1}(x, \xi) \cdot \widehat{\boldsymbol{\nu}}(\xi)\right|_{\xi \in \sigma_{0}} \leqslant 0$.

По лемме 4.3 из [5] для ограниченной липшицевой области $G$ и заданной функции $\alpha(\xi) \in L^{\infty}(\partial G)$ найдутся векторное поле $\boldsymbol{b}(\xi) \in L^{\infty}(G)^{n}$ и функция $q(\xi) \in L^{\infty}(G)$ такие, что соотношения $\operatorname{div}_{\xi} b=q$ в $G$ и $\boldsymbol{b} \cdot \nu_{\partial G}=\alpha$ на $\partial G$ выполняются в смысле интегрального тождества ${ }^{1}$

$$
\int_{\partial G} \alpha(\xi) \Phi(\xi) d S=\int_{G} \Phi(\xi) q(\xi) d \xi+\int_{G} \boldsymbol{b}(\xi) \cdot \nabla_{\xi} \Phi d \xi \quad \forall \Phi \in H^{1}(G) .
$$

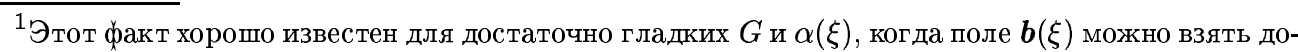
статочно гладким, $q=$ const, а указанные соотношения выполняются в классическом смысле.
} 
Пользуясь этим утверждением для $G=\omega_{\square}$ и взяв произвольную $\psi(\xi) \in L_{\mathrm{per}}^{\infty}(\partial \omega)$ такую, что $\psi(\xi) \geqslant 0$ на $\partial_{0} \omega, \psi(\xi)=0$ на $\partial \omega \backslash \partial_{0} \omega$, построим матрицу $\boldsymbol{B}(\xi) \in L_{\text {per }}^{\infty}(\omega)^{n \times n_{0}}$ (из $n$ столбцов и $n_{0}$ строк) и вектор-столбец $\boldsymbol{Q}(\xi) \in L^{\infty}(\omega)^{n_{0}}$, для которых

$$
\operatorname{div}_{\xi} \boldsymbol{B}=\boldsymbol{Q} \quad \text { в } \omega_{\square},\left.\quad \boldsymbol{B}(\xi) \boldsymbol{\nu}(\xi)\right|_{\sigma_{0}}=\psi(\xi) \widehat{\boldsymbol{\nu}}(\xi),\left.\quad \boldsymbol{B}(\xi) \boldsymbol{\nu}(\xi)\right|_{\partial \omega_{\square} \backslash \sigma_{0}}=\mathbf{0}
$$

в смысле интегрального тождества, являющегося матричным аналогом (17). Здесь $\operatorname{div}_{\xi} \boldsymbol{B}$ и $\boldsymbol{B} \boldsymbol{\nu}-$ векторы-столбцы размерности $n_{0}$ с компонентами $\partial B_{i j} / \partial \xi_{j}$ и $B_{i j} \nu_{j}$ соответственно. Из указанного интегрального тождества, периодически продолжив $\boldsymbol{B}$ и $\boldsymbol{Q}$ на $\omega$ и взяв любую $\varphi(x) \in C_{0}^{\infty}(\Omega), \varphi \geqslant 0$, получим

$$
\begin{aligned}
& \int_{S_{0}^{\varepsilon}} \varphi(x) \psi\left(\varepsilon^{-1} x\right) \boldsymbol{v}^{\varepsilon} \cdot \widehat{\boldsymbol{\nu}}\left(\varepsilon^{-1} x\right) d S_{x}=\int_{\Omega^{\varepsilon}} \varphi(x) \nabla_{x} \boldsymbol{v}^{\varepsilon}: \boldsymbol{B}\left(\varepsilon^{-1} x\right) d x \\
& +\varepsilon^{-1} \int_{\Omega^{\varepsilon}} \varphi(x) \boldsymbol{v}^{\varepsilon} \cdot \operatorname{div}_{\xi} \boldsymbol{B}\left(\varepsilon^{-1} x\right) d x+\int_{\Omega^{\varepsilon}} \boldsymbol{v}^{\varepsilon} \cdot \boldsymbol{B}\left(\varepsilon^{-1} x\right) \nabla_{x} \varphi d x
\end{aligned}
$$

где знак : означает скалярное произведение матриц $\boldsymbol{B}=\left\{B_{i j}\right\}$ и $\nabla \boldsymbol{v}^{\varepsilon}=\left\{\partial v_{i}^{\varepsilon} / x_{j}\right\}$ со столбцами $j=1, \ldots, n$ и строками $i=1, \ldots, n_{0}$.

Переходя в этом равенстве к пределу при $\varepsilon \rightarrow 0$ по построенной подпоследовательности, для которой вьполнено $(14)$, и учитьвая условие $\left.\boldsymbol{v}^{\varepsilon} \cdot \widehat{\boldsymbol{\nu}}\left(\varepsilon^{-1} x\right)\right|_{S_{0}^{\varepsilon}} \leqslant 0$, получим

$$
0 \geqslant \int_{\Omega \times \omega_{\square}} \varphi(x) \boldsymbol{B}(\xi): \nabla_{\xi} \boldsymbol{v}^{1}(x, \xi) d x d \xi+\int_{\Omega \times \omega_{\square}} \varphi(x) \boldsymbol{v}^{1} \cdot \operatorname{div}_{\xi} \boldsymbol{B}(\xi) d x d \xi
$$

Отсюда в силу упомянутого интегрального тождества типа (17) для $\boldsymbol{B}$ и $\boldsymbol{Q}=\operatorname{div}_{\xi} \boldsymbol{B}$ находим, что

$$
\int_{\Omega \times \sigma_{0}} \varphi(x) \psi(\xi) \boldsymbol{v}^{1}(x, \xi) \cdot \widehat{\boldsymbol{\nu}}(\xi) d x d S_{\xi} \leqslant 0 .
$$

Это означает, что $\left.\boldsymbol{v}^{1}(x, \xi) \cdot \widehat{\boldsymbol{\nu}}(\xi)\right|_{\xi \in \sigma_{0}} \leqslant 0$, так как $\varphi \geqslant 0, \psi \geqslant 0$ произвольны.

Для продолжения вектор-функций с области $\Omega^{\varepsilon}$ на не зависяший от $\varepsilon$ куб $Q \supset \bar{\Omega}$ будем использовать линейные операторы

$$
\begin{gathered}
P_{\varepsilon}: H_{0}^{1}\left(\Omega^{\varepsilon}, \Gamma^{\varepsilon}\right)^{n} \rightarrow H_{0}^{1}(Q)^{n}, \quad \sup _{\varepsilon}\left\|P_{\varepsilon}\right\|<\infty, \\
P_{\varepsilon} \boldsymbol{u}(x)=\mathbf{0} \quad \text { при п.в. } x \in Q \backslash \Omega, \text { для которых } \operatorname{dist}(x, \partial \Omega) \geqslant 4 \sqrt{n} \varepsilon .
\end{gathered}
$$

Кроме того, нам потребуется равномерное неравенство Корна

$$
\|\boldsymbol{v}\|_{1, \Omega^{\varepsilon}} \leqslant C\|\boldsymbol{e}(\boldsymbol{v})\|_{0, \Omega^{\varepsilon}} \quad \forall \boldsymbol{v} \in H_{0}^{1}\left(\Omega^{\varepsilon}, \Gamma^{\varepsilon}\right)^{n} .
$$

В случае гладких границ $\partial \Omega$ и $\partial \omega$ достаточно общей структуры существование операторов $P_{\varepsilon}$ и неравенство Корна установлены в [4, гл. 1 , теоремы $\left.4.3,4.5\right]$. В рассматриваемой ситуации, ког да $\Omega^{\varepsilon}, \Omega$ и $\left.\omega_{\square}=\omega \cap\right] 0,1\left[^{n}\right.$ - липшицевы области, эти факты нетрудно вывести из результатов $[5, \S 3]$ о продолжениях и неравенствах Корна. 
Введем следующие обозначения:

$$
\begin{gathered}
\mathcal{W}=\left\{\boldsymbol{w}(\xi) \in H_{\mathrm{per}}^{1}(\omega)^{n}:\left.\boldsymbol{w}(\xi) \cdot \boldsymbol{\nu}(\xi)\right|_{\sigma_{0}} \leqslant 0\right\}, \quad \mathbf{H}=H_{0}^{1}(\Omega)^{n} \times L^{2}(\Omega, \mathbb{H}), \\
\mathbb{H}=H_{\mathrm{per}}^{1}(\omega)^{n} / \mathbb{R}^{n}, \quad \mathbb{W}=\left\{\boldsymbol{W}(\xi) \in \mathbb{H}: \exists \boldsymbol{w} \in \mathcal{W}, \nabla_{\xi} \boldsymbol{w}=\nabla_{\xi} \boldsymbol{W}\right\}, \\
\mathfrak{L}^{2}(\Omega, \mathbb{W})=\left\{\boldsymbol{W}(x, \xi) \in L^{2}(\Omega, \mathbb{H}): \boldsymbol{W}(x, \cdot) \in \mathbb{W} \text { п.в. в } \Omega\right\} .
\end{gathered}
$$

Элементами пространства $\mathbf{H}$ являются пары вида $\left\{\boldsymbol{v}^{0}, \boldsymbol{v}^{1}\right\}=\left\{\boldsymbol{v}^{0}(x), \boldsymbol{v}^{1}(x, \xi)\right\}$, где $\boldsymbol{v}^{0}(x) \in H_{0}^{1}(\Omega)^{n}, \boldsymbol{v}^{1}(x, \xi) \in L^{2}(\Omega, \mathbb{H})$. Для этих элементов определим матрицы

$$
\boldsymbol{E}\left\{\boldsymbol{v}^{0}, \boldsymbol{v}^{1}\right\}=\boldsymbol{e}_{x}\left(\boldsymbol{v}^{0}\right)+\boldsymbol{e}_{\xi}\left(\boldsymbol{v}^{1}\right)
$$

Установим теорему об усреднении для задачи (3) с условиями Синьорини на $S_{0}^{\varepsilon}$, задающими двухсторонние препятствия по $n_{0}$ направлениям, $0<n_{0}<n$ (см. рис. 1 (b)). В этом случае

$$
\mathcal{M}^{0}=\left\{\boldsymbol{\eta}={ }^{t}(\widehat{\boldsymbol{\eta}}, \widetilde{\boldsymbol{\eta}}): \widehat{\boldsymbol{\eta}}=\widehat{\mathbf{0}} \in \mathbb{R}^{n_{0}}\right\}
$$

a

$$
\begin{gathered}
\mathcal{V}^{0}=\left\{\boldsymbol{v}={ }^{t}(\widehat{\mathbf{0}}, \widetilde{\boldsymbol{v}}) \in H_{0}^{1}(\Omega)^{n}\right\} \\
\mathcal{W}=\left\{\boldsymbol{w}(\xi)={ }^{t}(\widehat{\boldsymbol{w}}, \widetilde{\boldsymbol{w}}) \in H_{\mathrm{per}}^{1}(\omega)^{n}:\left.\widehat{\boldsymbol{w}}(\xi) \cdot \widehat{\boldsymbol{\nu}}(\xi)\right|_{\sigma_{0}} \leqslant 0\right\} .
\end{gathered}
$$

Теорема 1. Пусть $\boldsymbol{u}^{\varepsilon}-$ последовательность решений задачи (3) с условиями Синьорини на $S_{0}^{\varepsilon}$, задающими двухсторонние препятствия по $n_{0}$ направлениям, $0<n_{0}<n$. Тогда при $\varepsilon \rightarrow 0$ для решений, их градиентов и әнергий имеет место сходимость

$$
\begin{gathered}
P_{\varepsilon} \boldsymbol{u}^{\varepsilon} \stackrel{L^{2}(\Omega)}{\rightarrow} \boldsymbol{u}^{0}(x), \quad \nabla_{x} P_{\varepsilon} \boldsymbol{u}^{\varepsilon} \stackrel{2}{\rightarrow} \nabla_{x} \boldsymbol{u}^{0}(x)+\nabla_{\xi} \boldsymbol{u}^{1}(x, \xi), \\
\int_{\Omega^{\varepsilon}} \boldsymbol{e}\left(\boldsymbol{u}^{\varepsilon}\right): \mathcal{A}\left(\varepsilon^{-1} x\right) \boldsymbol{e}\left(\boldsymbol{u}^{\varepsilon}\right) d x \rightarrow \int_{\Omega \times \omega_{\square}} \boldsymbol{E}\left\{\boldsymbol{u}^{0}, \boldsymbol{u}^{1}\right\}: \mathcal{A}(\xi) \boldsymbol{E}\left\{\boldsymbol{u}^{0}, \boldsymbol{u}^{1}\right\} d x d \xi
\end{gathered}
$$

əде $\left.\left\{\boldsymbol{u}^{0}(x), \boldsymbol{u}^{1}(x, \xi)\right\}\right|_{\Omega \times \omega} \in \mathcal{V}^{0} \times \mathfrak{L}^{2}(\Omega, \mathbb{W})$ - единственное решение следующей задачи для вариационного неравенства: для любого әлемента $\left\{\boldsymbol{v}^{0}, \boldsymbol{v}^{1}\right\} \in \mathcal{V}^{0} \times \mathfrak{L}^{2}(\Omega, \mathbb{W})$

$$
\int_{\Omega \times \omega_{\square}} \boldsymbol{E}\left\{\boldsymbol{u}^{0}, \boldsymbol{u}^{1}\right\}: \mathcal{A}(\xi) \boldsymbol{E}\left\{\boldsymbol{v}^{0}-\boldsymbol{u}^{0}, \boldsymbol{v}^{1}-\boldsymbol{u}^{1}\right\} d x d \xi \geqslant\left|\omega_{\square}\right| \int_{\Omega} \boldsymbol{f} \cdot\left(\boldsymbol{v}^{0}-\boldsymbol{u}^{0}\right) d x .
$$

Решение $\left\{\boldsymbol{u}^{0}, \boldsymbol{u}^{1}\right\}$ имеет вид $\left\{\boldsymbol{u}^{0}(x), \boldsymbol{U}\left(\boldsymbol{e}_{\boldsymbol{x}}\left(\boldsymbol{u}^{0}\right), \xi\right)\right\}$, де $\boldsymbol{U}(\boldsymbol{p}, \xi) \in \mathbb{W}$ для любой постоянной матрицы $\boldsymbol{p} \in \mathbb{M}^{n}$ является единственным решением задачи на ячейке

$$
\int_{\omega_{\square}} \boldsymbol{e}_{\xi}(\boldsymbol{U}): \mathcal{A}(\xi) \boldsymbol{e}_{\xi}(\boldsymbol{V}-\boldsymbol{U}) d \xi \geqslant-\int_{\omega_{\square}} \boldsymbol{p}: \mathcal{A}(\xi) \boldsymbol{e}_{\xi}(\boldsymbol{V}-\boldsymbol{U}) d \xi \quad \forall \boldsymbol{V} \in \mathbb{W} .
$$

Кроме того, на $\boldsymbol{U}(\boldsymbol{p}, \xi)$ достигается

$$
\inf _{\boldsymbol{V} \in \mathbb{W}}\left\langle\left(\boldsymbol{p}+\boldsymbol{e}_{\xi}(\boldsymbol{V})\right): \mathcal{A}(\xi)\left(\boldsymbol{p}+\boldsymbol{e}_{\xi}(\boldsymbol{V})\right)\right\rangle_{\omega} \stackrel{\text { def }}{=} \mathbb{A}(\boldsymbol{p}) .
$$

Компонента $\boldsymbol{u}^{0}(x) \in \mathcal{V}^{0}$ является решением следующей задачи на минимум:

$$
\inf _{\boldsymbol{v}^{0} \in \mathcal{V}^{0}}\left\{\int_{\Omega} \mathbb{A}\left(\boldsymbol{e}_{x}\left(\boldsymbol{v}^{0}\right)\right) d x-2 \int_{\Omega} \boldsymbol{v}^{0} \cdot \boldsymbol{f} d x\right\} .
$$


ДокАЗАТЕЛЬСТво. Пользуясь (3), (1) и равномерньм неравенством Корна (19), получаем равномерную оценку $\left\|\boldsymbol{u}^{\varepsilon}\right\|_{1, \Omega^{\varepsilon}} \leqslant C\|\boldsymbol{f}\|_{0, \Omega}$. Учитьвая свойства операторов продолжения (18), неравенство (16) и лемму 1 , мы видим, что $P_{\varepsilon} \boldsymbol{u}^{\varepsilon}$ - ограниченная последовательность в $H^{1}(\Omega)^{n}$, из которой можно выделить подпоследовательность такую, что для некоторого $\left\{\boldsymbol{u}^{0}, \boldsymbol{u}^{1}\right\}$ имеет место (21), причем $\left.\left\{\boldsymbol{u}^{0}, \boldsymbol{u}^{1}\right\}\right|_{\Omega \times \omega} \in \mathcal{V}^{0} \times \mathcal{W}$. Покажем, что $\left.\left\{\boldsymbol{u}^{0}, \boldsymbol{u}^{1}\right\}\right|_{\Omega \times \omega}$ является решением задачи (23). Для этого запишем (3) в виде

$$
\int_{\Omega^{\varepsilon}} \boldsymbol{e}\left(\boldsymbol{u}^{\varepsilon}\right): \mathcal{A}^{\varepsilon} \boldsymbol{e}\left(\boldsymbol{v}^{\varepsilon}\right) d x-\int_{\Omega^{\varepsilon}} \boldsymbol{f} \cdot \boldsymbol{v}^{\varepsilon} d x \geqslant \int_{\Omega^{\varepsilon}} \boldsymbol{e}\left(\boldsymbol{u}^{\varepsilon}\right): \mathcal{A}^{\varepsilon} \boldsymbol{e}\left(\boldsymbol{u}^{\varepsilon}\right) d x-\int_{\Omega^{\varepsilon}} \boldsymbol{f} \cdot \boldsymbol{u}^{\varepsilon} d x
$$

и возьмем

$$
\begin{gathered}
\boldsymbol{v}^{\varepsilon}=\boldsymbol{v}^{0}(x)+\varepsilon \boldsymbol{v}^{1}\left(x, \varepsilon^{-1} x\right), \quad \boldsymbol{v}^{0} \in \mathcal{V}^{0}, \quad \boldsymbol{v}^{1}(y, \xi)=\sum_{s=1}^{m} \varphi_{s}(y) \boldsymbol{w}^{s}(\xi), \\
0 \leqslant \varphi_{s} \in C_{0}^{\infty}(\Omega), \quad \operatorname{supp} \varphi_{s} \subset \Omega_{s}, \quad \boldsymbol{w}^{s}(\xi) \in \mathcal{W}, \quad s=1, \ldots, m<\infty,
\end{gathered}
$$

где $\Omega_{s}$ - взаимно непересекающиеся липшицевы подобласти $\Omega$. Очевидно, что $\boldsymbol{v}^{\varepsilon} \in \mathcal{V}^{\varepsilon}$. Перейдем к пределу в (27) по найденной подпоследовательности (21), учитьвая при этом равенство $\boldsymbol{e}_{\boldsymbol{x}}\left(\boldsymbol{v}^{\varepsilon}(x)\right)=\boldsymbol{e}_{x}\left(\boldsymbol{v}^{0}\right)+\varepsilon \boldsymbol{e}_{y}\left(\boldsymbol{v}^{1}(y, \xi)\right)+\boldsymbol{e}_{\xi}\left(\boldsymbol{v}^{1}(y, \xi)\right)$ при $y=x, \xi=\varepsilon^{-1} x$, свойство среднего значения (12) и неравенство (13) при $\boldsymbol{V}^{\varepsilon}(x)=\boldsymbol{e}_{\boldsymbol{x}}\left(\boldsymbol{u}^{\varepsilon}\right), \boldsymbol{V}^{0}=\boldsymbol{e}_{\boldsymbol{x}}\left(\boldsymbol{u}^{0}\right)+$ $\boldsymbol{e}_{\xi}\left(\boldsymbol{u}^{1}\right), \boldsymbol{M}(\xi)=\chi_{\omega}(\xi) \mathcal{A}(\xi)$ (здесь $\chi_{\omega}(\xi)$ - характеристическая функция $\left.\omega\right)$. Получим

$$
\begin{aligned}
& \int_{\Omega \times \omega_{\square}}\left(\boldsymbol{e}_{x}\left(\boldsymbol{u}^{0}\right)+\boldsymbol{e}_{\xi}\left(\boldsymbol{u}^{1}\right)\right): \mathcal{A}(\xi)\left(\boldsymbol{e}_{x}\left(\boldsymbol{v}^{0}\right)+\boldsymbol{e}_{\xi}\left(\boldsymbol{v}^{1}(x, \xi)\right)\right) d x d \xi-\left|\omega_{\square}\right| \int_{\Omega} \boldsymbol{f} \cdot \boldsymbol{v}^{0} d x \\
& \geqslant \liminf _{\varepsilon \rightarrow 0}\left(\int_{\Omega^{\varepsilon}} \boldsymbol{e}\left(\boldsymbol{u}^{\varepsilon}\right): \mathcal{A}\left(\varepsilon^{-1} x\right) \boldsymbol{e}\left(\boldsymbol{u}^{\varepsilon}\right) d x-\int_{\Omega^{\varepsilon}} \boldsymbol{f} \cdot \boldsymbol{u}^{\varepsilon} d x\right) \\
& \geqslant \int_{\Omega \times \omega_{\square}}\left(\boldsymbol{e}_{x}\left(\boldsymbol{u}^{0}\right)+\boldsymbol{e}_{\xi}\left(\boldsymbol{u}^{1}\right)\right) \mathcal{A}(\xi)\left(\boldsymbol{e}_{x}\left(\boldsymbol{u}^{0}\right)+\boldsymbol{e}_{\xi}\left(\boldsymbol{u}^{1}\right)\right) d x d \xi-\left|\omega_{\square}\right| \int_{\Omega} \boldsymbol{f} \cdot \boldsymbol{u}^{0} d x
\end{aligned}
$$

Пользуясь неравенством (15), нетрудно проверить, что $\mathbb{W}$ есть замкнутый вьпуклый конус в $\mathbb{H}$. Можно также показать ${ }^{2}$, что $\mathfrak{L}^{2}(\Omega, \mathbb{W})$ есть замкнутый выпуклый конус в $L^{2}(\Omega, \mathbb{H})$, а функции $\boldsymbol{v}^{1}(x, \xi)$ вида $(28)$ всюду плотны в $\mathfrak{L}^{2}(\Omega, \mathbb{W})$. Следовательно, неравенство (29) вьполняется для любого элемента $\left\{\boldsymbol{v}^{0}(x), \boldsymbol{v}^{1}(x, \xi)\right\} \in \mathcal{V}^{0} \times \mathfrak{L}^{2}(\Omega, \mathbb{W})$, откуда и вытекает (23), а также сходимость энергий (22) (по подпоследовательности).

Покажем, что решение $\left\{\boldsymbol{u}^{0}, \boldsymbol{u}^{1}\right\}$ задачи (23) единственно. Для этого установим следующее неравенство типа Корна для элементов $\mathbf{H : ~}$

$$
\left\|\boldsymbol{v}^{0}\right\|_{1, \Omega}+\left\|\nabla_{\xi} \boldsymbol{v}^{1}\right\|_{0, \Omega \times \omega_{\square}} \leqslant C\left\|\boldsymbol{e}_{x}\left(\boldsymbol{v}^{0}\right)+\boldsymbol{e}_{\xi}\left(\boldsymbol{v}^{1}\right)\right\|_{0, \Omega \times \omega_{\square}} \quad \forall\left\{\boldsymbol{v}^{0}, \boldsymbol{v}^{1}\right\} \in \mathbf{H}
$$

\footnotetext{
${ }^{2}$ Для доказательства нужно рассмотреть соответствующие ступенчатые функции в $\Omega$ со значениями в $\mathbb{W}$ и воспользоваться тем, что среднее значение от функции, принимающей значения в замкнутом выпуклом множестве, принадлежит этому множеству; см. [10, гл. 4, $\S 4$, следствие 4 теоремы 39.]
} 
Воспользуемся неравенствами Корна (см. [4, гл. 1, теоремы 2.1 и 2.8])

$$
\begin{gathered}
\|\boldsymbol{v}\|_{1, \Omega} \leqslant C_{0}\left\|\boldsymbol{e}_{x}(\boldsymbol{v})\right\|_{0, \Omega} \quad \forall \boldsymbol{v} \in H_{0}^{1}(\Omega)^{n} \\
\left\|\boldsymbol{w}-\langle\boldsymbol{w}\rangle_{\omega}\right\|_{1, \omega_{\square}} \leqslant C_{1}\left\|\boldsymbol{e}_{\xi}(\boldsymbol{w})\right\|_{0, \omega_{\square}} \quad \forall \boldsymbol{w} \in H_{\mathrm{per}}^{1}(\omega)^{n} .
\end{gathered}
$$

Покажем, что найдется постоянная $C>0$, для которой выполнено неравенство

$$
|\boldsymbol{p}|^{2}+\int_{\omega_{\square}}\left|\boldsymbol{e}_{\xi}(\boldsymbol{w})\right|^{2} d \xi \leqslant C \int_{\omega_{\square}}\left|\boldsymbol{p}+\boldsymbol{e}_{\xi}(\boldsymbol{w})\right|^{2} d \xi \quad \forall \boldsymbol{p} \in \mathbb{S}^{n}, \quad \forall \boldsymbol{w} \in H_{\mathrm{per}}^{1}(\omega)^{n}
$$

Предположив противное, т.е. можем построить последовательности $\boldsymbol{p}^{m} \in \mathbb{S}^{n}$ и $\boldsymbol{w}^{m} \in$ $H_{\mathrm{per}}^{1}(\omega)^{n}$ такие, что $\boldsymbol{p}^{m} \rightarrow \boldsymbol{p}^{0} \in \mathbb{S}^{n}$ при $m \rightarrow \infty$ и

$$
\int_{\omega_{\square}}\left|\boldsymbol{p}^{m}+\boldsymbol{e}_{\xi}\left(\boldsymbol{w}^{m}\right)\right|^{2} d \xi \rightarrow 0, \quad\left|\boldsymbol{p}^{m}\right|^{2}+\int_{\omega_{\square}}\left|\boldsymbol{e}_{\xi}\left(\boldsymbol{w}^{m}\right)\right|^{2} d \xi=1, \quad\left\langle\boldsymbol{w}^{m}\right\rangle_{\omega}=0
$$

Поэтому

$$
\left\|\boldsymbol{p}^{0}+\boldsymbol{e}_{\xi}\left(\boldsymbol{w}^{m}\right)\right\|_{0, \omega_{\square}} \rightarrow 0
$$

откуда в силу (32) следует, что $\left\{\boldsymbol{w}^{m}\right\}$ - последовательность Коши в $H_{\text {per }}^{1}(\omega)^{n}$ и, значит, $\boldsymbol{w}^{m} \rightarrow \boldsymbol{w}^{0}$ для некоторого вектора $\boldsymbol{w}^{0} \in H_{\mathrm{per}}^{1}(\omega)^{n}$. Итак, учитьвая равенство $\boldsymbol{p}^{0}=\boldsymbol{e}_{\xi}\left(\boldsymbol{p}^{0} \boldsymbol{\xi}\right)$ при $\boldsymbol{\xi}={ }^{t} \xi$, получаем

$$
0=\int_{\omega_{\square}}\left|\boldsymbol{p}^{0}+\boldsymbol{e}_{\xi}\left(\boldsymbol{w}^{0}\right)\right|^{2} d \xi=\int_{\omega_{\square}}\left|\boldsymbol{e}_{\xi}\left(\boldsymbol{p}^{0} \boldsymbol{\xi}+\boldsymbol{w}^{0}\right)\right|^{2} d \xi
$$

Следовательно, имеем жесткое перемещение $\boldsymbol{p}^{0} \boldsymbol{\xi}+\boldsymbol{w}^{0}=\boldsymbol{Q} \boldsymbol{\xi}+\boldsymbol{b}$, т.е. $\boldsymbol{Q}$ - кососимметрическая матрица с постоянными элементами, a $\boldsymbol{b}$ - постоянный вектор. Пользуясь тем, что $\boldsymbol{w}^{0}$ периодична и имеет нулевое среднее значение, заключаем, что $\boldsymbol{w}^{0} \equiv \mathbf{0}$, а также $\boldsymbol{p}^{0}=\mathbf{0}$. Это противоречит (34), и, тем самым, неравенство (33) доказано.

Из (33) нетрудно вывести следующее неравенство для $\left\{\boldsymbol{v}^{0}, \boldsymbol{v}^{1}\right\} \in \mathbf{H}$ :

$$
\int_{\Omega}\left|\boldsymbol{e}_{x}\left(\boldsymbol{v}^{0}\right)\right|^{2} d x+\int_{\Omega \times \omega_{\square}}\left|\boldsymbol{e}_{\xi}\left(\boldsymbol{v}^{1}\right)\right|^{2} d x d \xi \leqslant C \int_{\Omega \times \omega_{\square}}\left|\boldsymbol{e}_{x}\left(\boldsymbol{v}^{0}\right)+\boldsymbol{e}_{\xi}\left(\boldsymbol{v}^{1}\right)\right|^{2} d x d \xi
$$

Отсюда, пользуясь неравенствами Корна (31), (32), получаем (30).

Теперь если $\left\{\boldsymbol{v}^{0}, \boldsymbol{v}^{1}\right\}$ - разность двух решений задачи (23), то очевидно, что

$$
\left\|\boldsymbol{E}\left\{\boldsymbol{v}^{0}, \boldsymbol{v}^{1}\right\}\right\|_{0, \Omega \times \omega_{\square}}=0
$$

а в силу $(30)$ и $\left\{\boldsymbol{v}^{0}, \boldsymbol{v}^{1}\right\}=\mathbf{0} \in \mathbf{H}$.

Итак, решение задачи (23) единственно, и если приведшие к (21) рассуждения применить к любой подпоследовательности $\boldsymbol{u}^{\varepsilon}$, можно заключить, что (21) имеет место для всей последовательности. 
Как упомянуто вьше, множество $\mathbb{W}$ является замкнутым выпуклым конусом в $\mathbb{H}$ и справедливо неравенство Корна (32). Поэтому минимизируемьй функционал в (25) коэрцитивен и, значит, задачи (24) и (25) имеют единственное решение $\boldsymbol{U}(\boldsymbol{p}, \xi)$ для любой постоянной матрицы $\boldsymbol{p} \in \mathbb{M}^{n}$ (см. [3, гл. 2]). Из (24) легко получаем оценку

$$
\left\|\boldsymbol{U}\left(\boldsymbol{p}^{1}, \xi\right)-\boldsymbol{U}\left(\boldsymbol{p}^{2}, \xi\right)\right\|_{\mathbb{H}} \leqslant C\left|\boldsymbol{p}^{1}-\boldsymbol{p}^{2}\right|
$$

которая означает, что $\boldsymbol{p} \mapsto \boldsymbol{U}(\boldsymbol{p}, \cdot)$ - непрерьвное по Липшицу отображение из $\mathbb{M}^{n}$ в $\mathbb{H}$.

Предположим, что матрица $\boldsymbol{p}$ в (24) зависит от $x \in \Omega$ как от параметра, причем $\boldsymbol{p}=\boldsymbol{p}(x) \in L^{2}\left(\Omega, \mathbb{M}^{n}\right)$. Тогда

$$
\boldsymbol{u}(x, \xi) \stackrel{\text { def }}{=} \boldsymbol{U}(\boldsymbol{p}(x), \xi) \in \mathfrak{L}^{2}(\Omega, \mathbb{W})
$$

Взяв при этом $\boldsymbol{v}=\boldsymbol{v}(x, \xi) \in \mathfrak{L}^{2}(\Omega, \mathbb{W})$ в (24) и интегрируя (24) по $x \in \Omega$, мы видим, что $\boldsymbol{u}=\boldsymbol{u}(x, \xi)=\boldsymbol{U}(\boldsymbol{p}(x), \xi)$ является решением следуюшей задачи: для заданной $\boldsymbol{p}=\boldsymbol{p}(x) \in L^{2}\left(\Omega, \mathbb{M}^{n}\right)$ найти $\boldsymbol{u} \in \mathfrak{L}^{2}(\Omega, \mathbb{W})$ такую, что для любого $\boldsymbol{v} \in \mathfrak{L}^{2}(\Omega, \mathbb{W})$

$$
\int_{\Omega \times \omega_{\square}} \boldsymbol{e}_{\xi}(\boldsymbol{u}): \mathcal{A}(\xi) \boldsymbol{e}_{\xi}(\boldsymbol{v}-\boldsymbol{u}) d x d \xi \geqslant-\int_{\Omega \times \omega_{\square}} \boldsymbol{p}(x): \mathcal{A}(\xi) \boldsymbol{e}_{\xi}(\boldsymbol{v}-\boldsymbol{u}) d x d \xi
$$

Нетрудно видеть, что эта задача имеет ровно одно решение и на нем достигается

$$
\inf _{\boldsymbol{v} \in \mathfrak{L}^{2}(\Omega, \mathbb{W})} \int_{\Omega \times \omega_{\square}}\left(\boldsymbol{e}_{\xi}(\boldsymbol{v})+\boldsymbol{p}(x)\right): \mathcal{A}(\xi)\left(\boldsymbol{e}_{\xi}(\boldsymbol{v})+\boldsymbol{p}(x)\right) d x d \xi
$$

Заметим, что решение $\left\{\boldsymbol{u}^{0}, \boldsymbol{u}^{1}\right\}$ задачи (23) является единственным элементом, на котором достигается минимум функционала

$J\left(\boldsymbol{v}^{0}, \boldsymbol{v}^{1}\right)=\int_{\Omega \times \omega_{\square}}\left(\boldsymbol{e}_{x}\left(\boldsymbol{v}^{0}\right)+\boldsymbol{e}_{\xi}\left(\boldsymbol{v}^{1}\right)\right): \mathcal{A}(\xi)\left(\boldsymbol{e}_{x}\left(\boldsymbol{v}^{0}\right)+\boldsymbol{e}_{\xi}\left(\boldsymbol{v}^{1}\right)\right) d x d \xi-2\left|\omega_{\square}\right| \int_{\Omega} \boldsymbol{v}^{0} \cdot \boldsymbol{f} d x$

на $\mathcal{V}^{0} \times \mathfrak{L}^{2}(\Omega, \mathbb{W})$. Пусть $I(\boldsymbol{p}, \boldsymbol{v})$ - минимизируемый интеграл в $(36)$, а $\boldsymbol{U}(\boldsymbol{p}, \xi)$ - решение задачи на ячейке (25). Тогда для любой $\left\{\boldsymbol{v}^{0}, \boldsymbol{v}^{1}\right\} \in \mathcal{V}^{0} \times \mathfrak{L}^{2}(\Omega, \mathbb{W})$ имеем

$$
J\left(\boldsymbol{v}^{0}, \boldsymbol{v}^{1}\right) \geqslant I\left(\boldsymbol{e}_{x}\left(\boldsymbol{v}^{0}(x)\right), \boldsymbol{U}\left(\boldsymbol{e}_{x}\left(\boldsymbol{v}^{0}\right), \xi\right)\right)-2\left|\omega_{\square}\right|\left(\boldsymbol{v}^{0}, \boldsymbol{f}\right)_{0, \Omega} \geqslant J\left(\boldsymbol{u}^{0}, \boldsymbol{u}^{1}\right)
$$

где первое неравенство имеет место, так как $\boldsymbol{u}(x, \xi)=\boldsymbol{U}\left(\boldsymbol{e}_{\boldsymbol{x}}\left(\boldsymbol{v}^{0}\right), \xi\right)$ является решением задачи на минимум (36) при $\boldsymbol{p}(x)=\boldsymbol{e}_{\boldsymbol{x}}\left(\boldsymbol{v}^{0}(x)\right)$; второе неравенство в (37) верно, поскольку на $\left\{\boldsymbol{u}^{0}, \boldsymbol{u}^{1}\right\}$ достигается минимум функционала $J\left(\boldsymbol{v}^{0}, \boldsymbol{v}^{1}\right)$. Из (37) очевидным образом вытекает, что $\left\{\boldsymbol{u}^{0}, \boldsymbol{u}^{1}\right\}=\left\{\boldsymbol{u}^{0}(x), \boldsymbol{U}\left(\boldsymbol{e}_{x}\left(\boldsymbol{u}^{0}\right), \xi\right)\right\}$, а $\boldsymbol{u}^{0} \in \mathcal{V}^{0}$ является единственньм решением следуюшей задачи на минимум:

$$
\inf _{\boldsymbol{v}^{0} \in \mathcal{V}^{0}}\left\{I\left(\boldsymbol{e}_{x}\left(\boldsymbol{v}^{0}(x)\right), \boldsymbol{U}\left(\boldsymbol{e}_{x}\left(\boldsymbol{v}^{0}\right), \xi\right)\right)-2\left|\omega_{\square}\right|\left(\boldsymbol{v}^{0}, \boldsymbol{f}\right)_{0, \Omega}\right\}
$$


причем в силу (25) при $\boldsymbol{p}=\boldsymbol{e}_{x}\left(\boldsymbol{v}^{0}\right)$ имеем

$$
I\left(\boldsymbol{e}_{x}\left(\boldsymbol{v}^{0}\right), \boldsymbol{U}\left(\boldsymbol{e}_{x}\left(\boldsymbol{v}^{0}\right), \xi\right)\right)=\left|\omega_{\square}\right| \int_{\Omega} \mathbb{A}\left(\boldsymbol{e}_{x}\left(\boldsymbol{v}^{0}\right)\right) d x
$$

Таким образом, $\boldsymbol{u}^{0}$ является единственным решением задачи на минимум (26).

Отметим, что мы фактически доказали существование и единственность решения усредненной задачи (26), не описывая в явном виде свойств усредненного лагранжиана $\mathbb{A}(\boldsymbol{p})$, имеющего вид (25). Нетрудно показать, что $\mathbb{A}(\boldsymbol{p})$ - вьпуклая функция $\boldsymbol{p}$, положительно однородная второго порядка (ср. с квадратичной формой (9), задающей усредненньй тензор упругости в случае $\left.\operatorname{dim} \mathcal{M}^{0}=n\right)$.

Вернемся теперь к классу краевых условий Синьорини на $S_{0}^{\varepsilon}$ с $n$ направлениями выхода, когда, как показано в [1], усредненная задача имеет вид (8). Этот класс можно несколько расширить, допустив не только случай $\operatorname{dim} \mathcal{M}^{0}=n($ как на рис. $1(\mathrm{a}))$, но и случай $0<\operatorname{dim} \mathcal{M}^{0}<n$, при условии, что $\mathcal{M}^{0}$ становится $n$-мерньм, если из $\partial_{0} \omega$ устранить бесконечно малую окрестность некоторого множества нулевой емкости на $\partial \omega$ (т.е. снять все краевые условия на соответствующей части $S_{0}^{\varepsilon}$ ). Пример таких краевых условий дан на рис. 1 (c), где условия Синьорини ставятся на полуокружности и, будучи сняты вблизи точки $A$, соответствуют $n$-мерному конусу малого раствора. Покажем, что в этом случае усредненная задача совпадает с (8). Сначала напомним некоторые факты относительно множеств нулевой емкости.

По определению, множество $\gamma \subset Q=\left\{\xi \in \mathbb{R}^{n}:|\xi|<R\right\}$ имеет нулевую емкость, сар $\gamma=0$, если

$$
\inf _{\varphi}\|\nabla \varphi\|_{0, Q}^{2}=0 \quad \text { для } \varphi \in C^{\infty}(\bar{Q}), \quad\|\varphi\|_{0, Q}=1, \quad \varphi \equiv 0 \text { в окрестности } \gamma .
$$

Нетрудно показать, что условие сар $\gamma=0$ эквивалентно наличию последовательности функций $\varphi_{\delta}(\xi)$ со следующими свойствами:

$$
\begin{gathered}
\psi_{\delta} \in C^{\infty}(\bar{Q}), \quad 0 \leqslant \psi_{\delta} \leqslant 1, \quad \psi_{\delta}(\xi)=0, \text { если } \operatorname{dist}(\xi, \gamma)<\delta, \\
\psi_{\delta} \rightarrow 1 \text { в } H^{1}(Q) \text { при } \delta \rightarrow 0 .
\end{gathered}
$$

Для ограниченной липшицевой области $G$ из этих соотношений легко следует, что если $\gamma \subset \partial G$ и сар $\gamma=0$, то

$$
\psi_{\delta} u \stackrel{H^{1}(G)}{\rightarrow} u \quad \text { при } \delta \rightarrow 0 \quad \forall u \in H^{1}(G) \cap L^{\infty}(G) .
$$

Как известно, нулевую емкость имеет липшицева поверхность $\gamma \subset \partial G$ меньшей размерности, чем $\partial G$, т.е. $\operatorname{dim} \gamma \leqslant n-2$. Если $\gamma$ - точка в $\mathbb{R}^{2}$, например $\gamma=\{0\}$, то можно взять

$$
\psi_{\delta}(\xi)=\varphi\left(\frac{\ln \left(\delta^{-1}|\xi|\right)}{\ln \left(1+\delta^{-1 / 2}\right)}\right),
$$

где $\varphi(t)$ - произвольная гладкая функция на $\mathbb{R}^{1}$ такая, что $\varphi \geqslant 0, \varphi(t)=0$ при $t \leqslant 0$, $\varphi(t)=1$ при $t \geqslant 1$. Нетрудно построить аналогичные функции $\psi_{\delta}$ для достаточно гладких поверхностей размерности $\leqslant n-2$. 
Tеорема 2. Пусть либо $\operatorname{dim} \mathcal{M}^{0}=n$, либо имеется множество $\gamma \subset \partial_{\square} \omega$, $\operatorname{cap} \gamma=0$, такое, что для любой достаточно малой $\delta$-окрестности $\gamma$, обозначаемой $\gamma_{\delta}$, и некоторых векторов $\boldsymbol{\alpha}^{0} \in \mathcal{M}^{0}, \boldsymbol{\eta}^{0} \in \mathbb{R}^{n}$, не зависящих от $\delta$, выполнены условия

$$
\begin{gathered}
\operatorname{dim} \mathcal{M}^{\delta}=n, \quad \mathcal{M}^{\delta}=\bigcap_{\xi \in \sigma_{0} \backslash \gamma_{\delta}} \mathcal{M}_{\xi}, \\
\boldsymbol{\alpha}^{0}+\rho \boldsymbol{B}_{\eta^{0}} \subset \mathcal{M}^{\delta} \quad \forall \rho \in\left[0, \rho_{\delta}\left[, \quad \boldsymbol{B}_{\eta^{0}}=\left\{\boldsymbol{\eta}:\left|\boldsymbol{\eta}^{0}-\boldsymbol{\eta}\right| \leqslant 1\right\}, \quad \rho_{\delta}>0 .\right.\right.
\end{gathered}
$$

Тогда выполнено утверждение теоремь 1 при $\mathcal{V}^{0}$ вида (6) $u \mathbb{W}=\mathbb{H}=H_{\mathrm{per}}^{1}(\omega)^{n} / \mathbb{R}^{n}$. При әтом $\boldsymbol{U}(\boldsymbol{p}, \xi)$ линейно зависит от $\boldsymbol{p} \in \mathbb{M}^{n}$, усредненный лагранэи иан $\mathbb{A}(\boldsymbol{p})$ совпадает с квадратичной формой (9) и задачу (26) можнн записать в виде (8) с тем же тензором $\widehat{\mathcal{A}}$. Таким образом, условия Синьорини на $S_{0}^{\varepsilon}$ влияют только на предельное множество допустимых перемещений $\mathcal{V}^{0}$, а не на усредненный тензор упругости.

ДокАЗАТЕЛЬСтво. Покажем, что при условиях теоремы множество $\mathbb{W}$ вида (20) всюду плотно в $\mathbb{H}$ (в отличие от $\mathbb{W}$ в теореме 1 , где оно было замкнутым подмножеством в $\mathbb{I})$.

Пусть $\Psi \in C_{\mathrm{per}}^{\infty}(\bar{\omega})^{n}$ и $C_{\Psi}=\sup _{\bar{\omega}}|\Psi|$. Из (41) вытекает, что для всех $\rho \in\left[0, \rho_{\delta}[\right.$ и всех $\xi \in \partial \omega$ имеем

$$
\boldsymbol{\alpha}^{0}+C_{\Psi}^{-1} \rho\left(C_{\Psi} \boldsymbol{\eta}^{0}+\boldsymbol{\Psi}(\xi)\right) \in \mathcal{M}^{\delta} .
$$

Поскольку сар $\gamma=0$, мы можем построить функции $\psi_{\delta}(\xi) \in C_{\mathrm{per}}^{\infty}(\bar{\omega})$, удовлетворяющие условиям $(39),(40)$ при $Q=\omega_{\square}=G$. Так как $\psi_{\delta}(\xi)$ принимает значения на $[0,1]$ и равна нулю в $\delta$-окрестности $\gamma$, то

$$
\boldsymbol{\alpha}^{0}+C_{\Psi}^{-1} \rho \psi_{\delta}(\xi)\left(C_{\Psi} \boldsymbol{\eta}^{0}+\boldsymbol{\Psi}(\xi)\right) \in \mathcal{M}^{0} \quad \text { для всех } \xi \in \partial \omega .
$$

Отсюда следует, что $\boldsymbol{\Psi}_{\delta}=C_{\Psi} \rho_{\delta}^{-1} \boldsymbol{\alpha}^{0}+\psi_{\delta}\left(C_{\Psi} \boldsymbol{\eta}^{0}+\boldsymbol{\Psi}\right) \in \mathcal{W}$ ввиду (7). В силу (40) имеем

$$
\left\|\nabla\left(\boldsymbol{\Psi}_{\delta}-\mathbf{\Psi}\right)\right\|_{0, \omega_{\square}}=\left\|\nabla\left(\left(1-\psi_{\delta}\right)\left(C_{\Psi} \boldsymbol{\eta}^{0}+\boldsymbol{\Psi}\right)\right)\right\|_{0, \omega_{\square}} \rightarrow 0 \quad \text { при } \delta \rightarrow 0 .
$$

Поскольку классы эквивалентности элементов $\Psi \in C_{\mathrm{per}}^{\infty}(\bar{\omega})^{n}$ плотны в $\mathbb{H}$, очевидно, что $\overline{\mathbb{W}}=\mathbb{H}$.

Далее доказательство проводится по схеме, использованной в теореме 1. Множество $\mathcal{V}^{0}$ имеет вид (6), поскольку для любой последовательности $\boldsymbol{v}^{\varepsilon} \in H^{1}(\Omega)^{n}$ такой, что $\boldsymbol{v}^{\varepsilon} \rightarrow \boldsymbol{v}^{0}$ в $H^{1}(\Omega)^{n}$, имеем сходимость

$$
\varepsilon \int_{S_{0}^{\varepsilon}}\left|\left(\boldsymbol{v}^{\varepsilon}, \boldsymbol{\nu}\left(\varepsilon^{-1} x\right)\right)^{+}\right|^{2} d S_{x} \rightarrow \int_{\Omega \times \sigma_{0}}\left|\left(\boldsymbol{v}^{0}, \boldsymbol{\nu}(\xi)\right)^{+}\right|^{2} d x d \xi
$$

В случае гладкой $\partial \omega$ довольно общего вида эта сходимость устанавливается точно так же, как лемма 4 в [11], а в рассматриваемом здесь случае липшицевой $\partial \omega$ ее нетрудно доказать на основе результатов $[5, \S 4]$. Линейная зависимость $\boldsymbol{U}(\boldsymbol{p}, \xi)$ от $\boldsymbol{p}$ и квадратическая структура лагранжиана $\mathbb{A}(\boldsymbol{p})$ вытекают из равенства $\overline{\mathbb{W}}=\mathbb{H}$. 
Заметим, что утверждение теоремы 1 , доказанноепри $0<n_{0}<n$, верно и при $n_{0}=n$ (условия Синьорини задают двухсторонние препятствия по всем направлениям), но в этом случае $\boldsymbol{u}^{0} \equiv \mathbf{0}, \boldsymbol{u}^{1} \equiv \mathbf{0}$. Тем не менее, и в этой ситуации можно доказать нетривиальньй результат об усреднении. Для этого в пространстве $L^{2}\left(\Omega, H_{\mathrm{per}}^{1}(\omega)^{n}\right)$ pacсмотрим замкнутьй вьпукльй конус $\mathfrak{L}^{2}(\Omega, \mathcal{W})$, состоящий из функций со значениями в множестве $\mathcal{W}$ из (20).

Теорема 3. Пусть $\boldsymbol{u}^{\varepsilon}(x)$ - решение задачи (3) с условиями Синьорини на $S_{0}^{\varepsilon}$, задающими двухсторонние препятствия по всем $n$ направлениям. Тогда

$$
\begin{gathered}
\left\|\boldsymbol{u}^{\varepsilon}\right\|_{0, \Omega^{\varepsilon}} \leqslant C_{0} \varepsilon^{2}, \quad\left\|\nabla \boldsymbol{u}^{\varepsilon}\right\|_{0, \Omega^{\varepsilon}} \leqslant C_{1} \varepsilon \\
\varepsilon^{-2} P_{\varepsilon} \boldsymbol{u}^{\varepsilon} \stackrel{2}{\rightarrow} \boldsymbol{u}^{1}(x, \xi) \quad(\text { по подпоследовательности }), \quad \varepsilon^{-1} \nabla P_{\varepsilon} \boldsymbol{u}^{\varepsilon} \stackrel{2}{\rightarrow} \nabla_{\xi} \boldsymbol{u}^{1}(x, \xi),
\end{gathered}
$$

$$
\frac{1}{\varepsilon^{2}} \int_{\Omega^{\varepsilon}} \boldsymbol{e}\left(\boldsymbol{u}^{\varepsilon}\right): \mathcal{A}\left(\varepsilon^{-1} x\right) \boldsymbol{e}\left(\boldsymbol{u}^{\varepsilon}\right) d x \rightarrow \int_{\Omega \times \omega_{\square}} \boldsymbol{e}_{\xi}\left(\boldsymbol{u}^{1}\right): \mathcal{A}(\xi) \boldsymbol{e}_{\xi}\left(\boldsymbol{u}^{1}\right) d x d \xi
$$

где $\left.\boldsymbol{u}^{1}(x, \xi)\right|_{\Omega \times \omega} \in \mathfrak{L}^{2}(\Omega, \mathcal{W})$ - решение следующей задачи: для любого $\boldsymbol{v}(x, \xi) \in$ $\mathfrak{L}^{2}(\Omega, \mathcal{W})$

$$
\int_{\Omega \times \omega_{\square}} \boldsymbol{e}_{\xi}\left(\boldsymbol{u}^{1}\right): \mathcal{A}(\xi) \boldsymbol{e}_{\xi}\left(\boldsymbol{v}-\boldsymbol{u}^{1}\right) d x d \xi \geqslant \int_{\Omega \times \omega_{\square}} \boldsymbol{f}(x) \cdot\left(\boldsymbol{v}-\boldsymbol{u}^{1}\right) d x d \xi
$$

Если решение $\boldsymbol{u}^{1}(x, \xi)$ задачи (45) единственно, то сходимость (43) для $\varepsilon^{-2} P_{\varepsilon} \boldsymbol{u}^{\varepsilon}$ имеет место для всей последовательности; в противном случае предельные решения $\boldsymbol{u}^{1}(x, \xi)$ и $\widetilde{\boldsymbol{u}}^{1}(x, \xi)$ отличаются слагаемым $\boldsymbol{\eta}(x) \in L^{2}(\Omega)^{n}$, для которого $(\boldsymbol{\eta}, \boldsymbol{f})_{L^{2}(\Omega)^{n}}=0 u \widetilde{\boldsymbol{u}}^{1}(x, \xi)=\boldsymbol{u}^{1}(x, \xi)+\boldsymbol{\eta}(x)$.

ДокАЗАТЕЛЬСТво. Из (3), (19), (16) и леммы 1 выводим равномерные оценки (42) и сходимости (43) для подпоследовательности и некоторого $\boldsymbol{u}^{1}(x, \xi) \in \mathfrak{L}^{2}(\Omega, \mathcal{W})$. Далее поступаем, слегка изменив схему, использованную в доказательстве теоремы 1 . А именно, записав неравенство (3) в виде (27) и разделив его на $\varepsilon^{2}$, возьмем $\boldsymbol{v}^{\varepsilon}=\varepsilon^{2} \boldsymbol{v}^{1}\left(x, \varepsilon^{-1} x\right) \in$ $\mathcal{V}^{\varepsilon}$, где $\boldsymbol{v}^{1}(x, \xi)$ того же вида, что в $(28)$. Перейдем в получившемся неравенстве к пределу по построенной подпоследовательности, учитьвая (43), (42) и (13) при $\boldsymbol{V}^{\varepsilon}=\varepsilon^{-1} \times$ $\boldsymbol{e}_{\boldsymbol{x}}\left(\boldsymbol{u}^{\varepsilon}\right), \boldsymbol{V}^{0}=\boldsymbol{e}_{\xi}\left(\boldsymbol{u}^{1}\right)$. Получим

$$
\begin{gathered}
\int_{\Omega \times \omega_{\square}} \boldsymbol{e}_{\xi}\left(\boldsymbol{u}^{1}(x, \xi)\right): \mathcal{A}(\xi) \boldsymbol{e}_{\xi}\left(\boldsymbol{v}^{1}(x, \xi)\right) d x d \xi-\int_{\Omega \times \omega_{\square}} \boldsymbol{f} \cdot \boldsymbol{v}^{1}(x, \xi) d x d \xi \\
\geqslant \liminf _{\varepsilon \rightarrow 0}\left(\frac{1}{\varepsilon^{2}} \int_{\Omega^{\varepsilon}} \boldsymbol{e}\left(\boldsymbol{u}^{\varepsilon}\right): \mathcal{A}\left(\varepsilon^{-1} x\right) \boldsymbol{e}\left(\boldsymbol{u}^{\varepsilon}\right) d x-\frac{1}{\varepsilon^{2}} \int_{\Omega^{\varepsilon}} \boldsymbol{f} \cdot \boldsymbol{u}^{\varepsilon} d x\right) \\
\geqslant \int_{\Omega \times \omega_{\square}} \boldsymbol{e}_{\xi}\left(\boldsymbol{u}^{1}\right): \mathcal{A}(\xi) \boldsymbol{e}_{\xi}\left(\boldsymbol{u}^{1}\right) d x d \xi-\int_{\Omega \times \omega_{\square}} \boldsymbol{f} \cdot \boldsymbol{u}^{1}(x, \xi) d x d \xi
\end{gathered}
$$

Поскольку конечные суммы $\boldsymbol{v}^{1}(x, \xi)$ образуют всюду плотноемножество в $\mathfrak{L}^{2}(\Omega, \mathcal{W})$, это неравенство верно для любой $v^{1}(x, \xi) \in \mathfrak{L}^{2}(\Omega, \mathcal{W})$. Отсюда следует, что $\boldsymbol{u}^{1}(x, \xi)-$ решение задачи (45) и имеет место сходимость энергий (44). Если $\boldsymbol{u}^{1}(x, \xi)$ - единственное 
решение, то очевидно, что доказанные сходимости имеют место для всей последовательности. Если же задача имеет несколько решений, нетрудно показать, что любое из них имеет вид суммы $\boldsymbol{u}^{1}(x, \xi)$ и вектора $\boldsymbol{\eta}(x) \in L^{2}(\Omega)^{n}$, для которого $(\boldsymbol{f}, \boldsymbol{\eta})_{L^{2}(\Omega)^{n}}=0$.

Некоторые другие задачи усреднения для упругих тел с нелинейньпи краевыми условиями (типа трения или “живых сил” на изрезанной или перфорированной части гранищы) рассмотрены в работах [1], [5], [11], [12].

\section{СПИСОК ЦИТИРОВАННОЙ ЛИТЕРАТУРЫ}

[1] Yosifian G.A. Some homogenization problems for the system of elasticity with nonlinear boundary conditions in perforated domains // Appl. Anal. 1999. V. 71. P. 379-411.

[2] Дюво Г., Лионс ЖК.-Л. Неравенства в механике и физике. М.: Наука, 1980.

[3] Экланд И., Темам Р. Выпуклый анализ и вариационные проблемы. М.: Мир, 1979.

[4] Олейник О. А., Иосифьян Г. А., Шамаев А. С. Математические задачи теории силно неоднородных упругих сред. М.: Изд-во МГУ, 1990.

[5] Иосифьян Г. А. О некоторых односторонних краевых задачах для упругих тел с изрезанной границей // Тр. семинара И. Г. Петровского. 1999. Т. 21. С. 240-297; (см. также Preprint № 99-18 (SFB 359) IWR, http://www.iwr.uni-heidelberg.de.) Univ. Heidelberg, 1999.

[6] Жиков В. В., Козлов С. М., Олейник О. А. Усреднение дифференциальных операторов и интегральных функционалов. М.: Физматгиз, 1993.

[7] Nguetseng G. A general convergence result for a functional related to homogenization // SIAM J. Math. Anal. 1980. V. 20. P. 608-623.

[8] Allaire G. Homogenization and two-scale convergence // SIAM J. Math. Anal. 1992. V. 23. P. 1482-1518.

[9] Жиков В.В.Об одном расширении и применении метода двухмасштабной сходимости // Матем. сб. 2000. Т. 191. № 7. С. 31-72.

[10] Шварц Л. Анализ. М.: Мир, 1972.

[11] Yosifian G. A. On some homogenization problems in perforated domains with nonlinear boundary conditions // Appl. Anal. 1997. V. 65. P. 257-288.

[12] Yosifian G. A. Homogenization of some contact problems for the system of elasticity in perforated domains // Rend. Sem. Mat. Univ. Padova. 2001. V. 105. P. 37-64.

Институт проблем механики РАН

Поступило

E-mail: yosifian@mtu-net.ru

14.01.2002 